\title{
Early Post Stroke Rehabilitation Load Correlates With Greater Functional Improvement of Hemiparesis
}

\author{
Caterina Sottini, ${ }^{1}$ Daniela Vangi, ${ }^{1}$ Emanuela Rizzi, ${ }^{1}$ Chiara Stampatori, ${ }^{1}$ and Flavia Mattioli ${ }^{1,}$ \\ ${ }^{1}$ Rehabilitation Unit, Spedali Civili of Brescia, Italy \\ "Corresponding author: Flavia Mattioli, Spedali Civili of Brescia, 25123 BRESCIA, Italy. Tel: +39-0302027218, E-mail: flaviacaterina.mattioli@gmail.com
}

Received 2016 May 10; Revised 2016 August 26; Accepted 2016 September 25.

\begin{abstract}
Background: Evidences show that generally stroke patients receiving greater amount of physiotherapy obtain better motor function over time, although this has not been investigated in the early post stroke population. The aim of the present observational retrospective study was to investigate the relationship between the amount of physiotherapy provided to early post acute stroke patients and their motor improvement.

Methods: Overall, 846 early stroke patients with hemiparesis, treated with physiotherapy, were retrospectively analyzed. Clinical data at admission as well as functional scales at the first evaluation and at discharge were analyzed. For each patient, a rehabilitation load (resulting from the total number of the 30' daily consecutive physiotherapy sessions they received) was used as the dose of physiotherapy. Pre and post rehabilitation scales were analyzed and their change $(\Delta)$ was correlated with the rehabilitation load. Results: The mean rehabilitation load was ten sessions (range 2 to 56). The scores of Barthel index, Motor Assessment Score, Motricity index for both lower and upper limb, Functional Ambulation Classification, Berg Balance scale and Trunk control test significantly improved from pre to post rehabilitation, and a significant correlation was found between the rehabilitation load and the improvement in Barthel index $(\mathrm{P}<0.05)$, Motor Assessment Score, Motricity Index for upper and lower limb $(\mathrm{P}<0.001)$, Berg Balance scale $(\mathrm{P}<0.05)$ and Trunk control test $(\mathrm{P}<0.001)$.

Conclusions: In this study, a significant correlation was found between early post stroke motor rehabilitation dose and greater improvement in functional scales, assessing patient's independence, balance and paretic limb's motricity.
\end{abstract}

Keywords: Stroke, Rehabilitation, Hemiparesis

\section{Background}

In western countries, stroke represents the third cause of death, the second cause of dementia and the first cause of severe loss of independence. Although stroke incidence and stroke-related death were reported to have decreased, longitudinal studies show an increase in the absolute number of strokes and of the stroke impact on disability in the world. Approximately 800,000 people in the USA have their first or recurrent stroke each year. The prevalence of chronic stroke in the USA is estimated at about seven million1, with about $80 \%$ of patients with stroke being over the age of 65 . The prevalence of stroke is likely to increase in the future due to the aging population. Even though acute stroke care has improved, for example by large-scale application of recombinant tissue plasminogen activator (rTPA) $(1,2)$ and organized interdisciplinary inpatient stroke care (3) (Stroke Units in Italy), a large number of patients still remain disabled regardless of the time that has elapsed post stroke.

Only $12 \%$ of the patients with stroke are independent in basic Activities of Daily Living (ADL) at the end of the first post stroke week (4). In the long term, 25 to $74 \%$ of patients have to rely on human assistance for basic ADLs, like feeding, self-care and mobility (5). In Italy ischemic stroke was reported in $80 \%$ of cases in the whole stroke population, whereas hemorrhagic stroke was diagnosed in $20 \%$. Stroke incidence was $6.5 \%$ in the general population, respectively 7.4\% in males and 5.9\% in females (6).

Multidisciplinary rehabilitation of stroke is recommended as the gold standard by several authors $(7,8)$ and better outcomes in patients, who underwent more intense motor rehabilitation regimens, than in patients, who received lower intensity ones have been reported, based on data taken from several randomized clinical trials, usually conducted on mixed acute and chronic large post stroke patients' populations, including patients submitted to several different rehabilitative therapies (9). To the best of our knowledge, the role of different amounts of physiotherapy in specific timeframes after stroke has not been exhaustively investigated till now, although early post stroke rehabilitation is recommended and prescribed worldwide. Pathophysiological mechanisms underlying motor recovery after stroke are supposedly different between acute post stroke and the chronic phase (10), consequently different effects are expected to be obtained by

Copyright @ $)$ 2016, Tehran University of Medical Sciences. This is an open-access article distributed under the terms of the Creative Commons Attribution-NonCommercial 4.0 International License (http://creativecommons.org/licenses/by-nc/4.0/) which permits copy and redistribute the material just in noncommercial usages, provided the original work is properly cited. 
physiotherapy in different post stroke periods.

In Italy, stroke patients, usually admitted to the Stroke Unit for acute care including thrombolysis, are referred to rehabilitation units, as soon as the critical conditions have been managed and the diagnostic procedures are concluded. Afterwards, they are often transferred to Rehabilitation Units until recovery is completed. In nearly all the Italian Stroke Units, early physiotherapy is started for patients, who present motor deficits and then are prosecuted in the rehabilitation units or (in milder cases) in outpatient settings. Their functional evaluation at admission in and at discharge from the Stroke Unit is frequently performed, including several scales measuring, both daily living patient's abilities and independence, ambulation quality or motor functions. Obviously, only a limited number of these patients appear to be completely recovered at the time of discharge from the Stroke Unit and do not need further rehabilitation, the majority of them need to be treated at specific rehabilitation units.

Although the effects of rehabilitation on motor recovery have been studied in several researches on patients with post stroke time of more than 10 to 30 days (9), to the best of our knowledge, the effect of early post stroke rehabilitation, as offered in the Stroke Unit, on functional independence and motor abilities of patients has not been investigated till now. This has both clinical and costeffectiveness implications, considering the allocation of rehabilitative resources within acute care hospitals (11).

This real life retrospective study was conducted on patients with hemiparesis/hemiplegia due to stroke admitted to the stroke unit of the Spedali Civili of Brescia from January 2012 to May 2015, to evaluate the correlation between the dose of physiotherapy provided in the early post stroke phase and different functional scales. We also investigated, on the basis of these results, which scales revealed to be the most useful to be used to detect functional motor improvement early after the stroke.

\section{Methods}

Clinical records of patients admitted to the Stroke Unit of Spedali Civili between January 2012 and May 2015, due to hemiparesis or hemiplegia as a result of stroke, who received early post stroke motor rehabilitation, were retrospectively considered for this study. Clinical and demographic characteristics were recorded, including gender, age, previous strokes, ischemic or hemorrhagic nature of the present stroke, the type of respiration (spontaneous or not) and the presence of dysphagia at admission. The Comitato Etico of the Spedali Civili authorized the study, according to good clinical practice rules. The national institute of health (NIH) Stroke Scale to evaluate stroke sever- ity at admission and at discharge was available, as well as the Rankin scale (12), for a generic evaluation of disability before and after the stroke (ranging from 0 (no symptoms) to 6 (dead)).

Time from admission to the first rehabilitative evaluation (where physiotherapy was prescribed and started) was calculated; all the patients were evaluated within 24 hours from admission to the Hospital and at discharge, by the physiatrist.

A motor rehabilitation load, quantifying the amount of physiotherapy provided to individual patients, was also calculated as the total number of consecutive physiotherapies per patient, assuming that each of them lasted 30' and was conducted daily by the physiotherapist and ended the day of patient's discharge. Discharge was organized independently from motor recovery whenever the critical conditions were overcome, thus it was possible for patients with more severe clinical condition to spend more time at the Stroke Unit, receiving more physiotherapy sessions. Patients, if needed also received speech therapy, but data on speech or swallowing disorders were not available so they were not considered.

Motor rehabilitation was carried on an individual basis by expert physiotherapists, which aimed at improving the functional movements of the patient with gradualism, according to the general status of the patient in the early post stroke phase and his/her stroke severity. In general, the objective of this early phase rehabilitation was helping the patients in moving from bed to sitting position, then maintaining the trunk control before moving from sitting to standing. For patients with complete plegia of the limbs, only passive assisted movements of the limbs were performed. On the other hand, mild paretic patients were treated with the aim of improving the strength and mobility of the limbs, with rehabilitation of gait as well as upper limb/hand fine movements. Balance of trunk and gait was also treated on an individual basis. A gradual progressive approach was used in order to avoid discomfort or pain in the patient and to stimulate proprioceptive sensibility and active limbs movements.

Patients were evaluated by using the following scales, which have been used to compare pre and post treatment status: 1) Barthel index (13), to assess independence in selfcare on a more specific level. For each item, the patient is given a specific score $(10=$ independent; $5=$ needs help $0=$ unable) depending of the grade of autonomy in self care; 2) Motor Assessment Score (MAS) (14) to measure patient's paretic limbs' movements and muscle tone scoring from 1 (worse performance) to 6 (best performance) on a total of 9 items (ability to move from supine to both sided position in the bed, from bed to sitting position, to maintain the sitting position, to move from sitting to standing, to 
walk, to use arm and hand, and finally the muscle tone), with the score of General Tonus ranging from 1 to 6 , increasing from hypotonus to hypertonus, where 4 indicates consistently normal tonus; 3 ) motricity index (15), to investigate fine movements of both left and right superior and inferior limbs in more detail at a functional level. The scale goes from 0 (complete paresis) to 100 (normal strength), and the score is obtained by summing subscores for different aspects of limb's movement; 4) Functional Ambulation Classification (FAC) (16) to assess qualitative aspects of ambulation, on a six point scale assessing how much human support the patient requires when walking, regardless of whether or not he/she needs a personal device; 5) Trunk control test (17), scoring patient's ability to turn on the affected and non affected side, to move from lying to sitting position and to maintain the sitting position from 0 to 25 ; 6) Berg balance scale (BBS)V (18), a 14-item scale designed to measure balance in different tasks, each item ranging from 0 (lowest level of function) to 4 (highest level of function).

\subsection{Statistical Analyses}

Simple parametric analyses were performed (means and standard deviations; comparison between pre and post treatment by using the Wilcoxon signed Rank test), and a correlation with Pearson method was performed in order to investigate correlations between the amount of treatment (the rehabilitation load) and the mean changes (delta: $\Delta$ ) in functional scales from pre to post rehabilitation.

\section{Results}

Overall, 846 patients were considered, of whom 465 were male and 381 female. Their mean NIH Stroke Scale was eight at admission and 6.4 at discharge. Furthermore, 713 patients out of 846 were admitted to the hospital due to their first stroke (84\%), the remaining had previous vascular accidents. Regarding the type of stroke, 640 were ischemic stroke and 194 hemorrhagic stroke (for 13 patients the data were missing); 365 had a left-sided hemiparesis and 481 a right-sided hemiparesis. The majority of the patients did not need respiratory assistance (731/846, 86\%) and 255 out of 846 (30\%) were dysphagic at the time of the first rehabilitative visit, when physiotherapy was prescribed and started.

Mean time elapsed from admission to pre rehabilitation assessment and starting of rehabilitation was 2.52 days, mean and standard deviation (SD) of rehabilitation load were 10 sessions and 2.8 (see Table 1 ), respectively, with ten days mean interval between pre and post rehabilitation evaluations.
Table 1. Clinical Characteristics of the Stroke Patients (N 846)

\begin{tabular}{lc}
\hline Characteristics & Values $^{\mathbf{a}}$ \\
\hline Age, $\mathbf{y}$ & $70.67 \pm 13.16$ \\
\hline Gender & 465 \\
\hline \multicolumn{1}{|c}{ Male } & 381 \\
\hline \multicolumn{1}{|c}{ Female } & $713(84)$ \\
\hline First event & $731(86)$ \\
\hline Normal breathing & 8 \\
\hline NIHSS & 6.4 \\
\hline \multicolumn{1}{c}{ Admission } & $2.52(2.8)$ \\
\hline \multicolumn{1}{c}{ Discharge } & $10.01 \pm 5.89$ \\
\hline Days from admission to1 ${ }^{\circ}$ evaluation (No., SD) & \\
\hline Rehabilitation load: number of daily 30' sessions & \\
\hline a Values are expressed as mean \pm SD or No. (\%). &
\end{tabular}

One hundred and thirty out of 846 patients were considered recovered at discharge, the remaining still needed physiotherapy, as such they were sent to rehabilitation units or to out patient rehabilitation.

A statistically significant worsening in the Ranking Scale before stroke compared to discharge after stroke was noticed, indicating a reduction in functional independence in the early post stroke phase (750 patients; $\mathrm{P}=$ 0.000). Means pre rehabilitation, post rehabilitation and changes (post - pre rehabilitation score: $\Delta$ ) in each functional scale are presented in Table 2. A statistically significant improvement was observed in motor assessment score (723 patients; $\mathrm{P}=0.000$ ), functional ambulation classification (764 patients; $\mathrm{P}=0.000$ ), Barthel index (768 patients; $\mathrm{P}=0.000)$, movement of the superior limb (613 patients; $\mathrm{P}=0.009$ ) and movement of the Inferior Limb in the motricity index scale (608 patients; $\mathrm{P}=0.000)$, trunk control test (756 patients; $\mathrm{P}=0.000$ ) and BBS (343 patients; $\mathrm{P}=$ $0.000)$, showing a general significant improvement in all these scales, at the end of treatment when the patient was discharged from the stroke unit.

Pearson correlation test between the rehabilitation load and the $\Delta$ scores in each functional scale revealed a significant positive correlation between rehabilitation load and Motricity indexes of both superior and inferior limb movements $(\mathrm{P}=0.000$ and $\mathrm{P}=0.009$, respectively), as well as between rehabilitation load and the Barthel index $(\mathrm{P}=0.002)$, motor assessment scale $(\Delta=0.000)$, BBS $(\mathrm{P}=0.002)$ and Trunk control test $(\mathrm{P}=0.000)$, suggesting a positive correlation between the load of treatment in the early post stroke phase and the improvement in independence measures, which were motor scales investigating ei- 
Table 2. Mean Pre Rehabilitation, Post Rehabilitation Scores (With Each Standard Deviation Score Written in Parenthesis) and Changes (post-pre $\Delta$ ) in Scores of Rankin, Barthel Index, MAS, Motricity Scale Upper and Lower Limb, BBS, Trunk Control Test and FAC

\begin{tabular}{|c|c|c|c|c|}
\hline & M PRE (SD) & M POST (SD) & $\Delta$ & PValue $^{\mathrm{a}}$ \\
\hline Rankin $^{b}$ & $3.93(1.18)$ & $3.46(1.41)$ & -0.47 & 0.00 \\
\hline MAS & $21.41(17.43)$ & $25.64(17.77)$ & 4.22 & 0.00 \\
\hline FAC & $1.15(1.61)$ & $1.71(1.93)$ & 0.56 & 0.00 \\
\hline Barthel & $34.96(33.93)$ & $43.47(36.71)$ & 8.5 & 0.00 \\
\hline Motricity lower limb & $59.86(35.25)$ & $63.19(34.87)$ & 3.32 & 0.00 \\
\hline BBS & $24.10(22.38)$ & $29.21(22.74)$ & & 0.00 \\
\hline Trunk Control Terst & $52.79(41.64)$ & $64,69(39.95)$ & & 0.00 \\
\hline
\end{tabular}

${ }^{\mathrm{a}}$ T Test P Value.

${ }^{\mathrm{b}}$ Pre stroke Rankin vs. Ranking at discharge.

ther changes in motricity of the paretic limbs or balance and trunk control.

On the other hand, no significant correlation was found between the rehabilitation load and the changes in functional ambulation $(\mathrm{P}=0.297)$ and Rankin $(\mathrm{P}=0.736)$ scales (Table 3).

Table 3. Pearson Correlation Results Between Rehabilitation Load and $\Delta$ Scores

\begin{tabular}{lcc}
\hline & r & P Value \\
\hline$\Delta$ Rankin & -0.012 & 0.736 \\
\hline$\Delta$ MAS & 0.130 & 0.000 \\
\hline$\Delta$ FAC & 0.037 & 0.297 \\
\hline$\Delta$ Barthel Index & 0.110 & 0.002 \\
\hline$\Delta$ Motricity upper limb & 0.092 & 0.009 \\
\hline$\Delta$ Motricity lower limb & 0.146 & 0.000 \\
\hline$\Delta$ BBS & 0.164 & 0.000 \\
\hline$\Delta$ Trunk Control Test & 0.179 & 0.000 \\
\hline
\end{tabular}

\section{Discussion}

The main result of this real life observational retrospective study was a dose-response relationship between the number of early post stroke physiotherapy sessions and the improvement in scales measuring both patients' independence in daily activities and the severity of hemiplegia, at the time of patients' discharge from the Stroke Unit. On average, patients received ten 30' daily physiotherapies and greater improvement was found in scales measuring upper and lower limb motility as well as measures of personal independence and balance. Of course the improve- ment in motor functions may be ascribed to the combination effect of both spontaneous recovery of hemiplegia and the role of physiotherapy, although, the effects of each of these two factors are hardily separable. The relevant result of this study was the significant correlation found between the load of motor rehabilitation given to patients in the acute post stroke phase and the greater amount of functional improvement in most functional scales, confirming the dose effect data observed in longer follow ups (9). The scales, which resulted to significantly correlate with the physiotherapy dose were Barthel index, measuring the independence in feeding, dressing, personal hygiene and moving from bed to chair and more specific scales addressing limbs motricity (MAS and Motricity indices of upper and lower limb) as well as balance (Trunk control test and BBS). On the other hand, FAC, measuring fine aspects of ambulation, did not correlate significantly with the rehabilitation load. The possible explanation of this is, in our opinion, that although it significantly improved from admission to discharge, ambulation is not frequently rehabilitated early after stroke, whereas physiotherapic treatment more frequently treats specifically limbs' movements and balance. The qualitative aspects of gait are typically treated later on, when the patients are stable in the standing position and have milder strength deficits in the paretic leg. Possibly, longer follow up of months would be able to detect significant effects of rehabilitation on ambulation improvements, but this necessarily implies the availability of patients' records afterwards, which was not the case in our study. The motor aspects, which are shown to significantly improve in the first week post stroke, are postural and balance changes (moving from bed to sitting position and from sitting to standing position as well as trunk balance) and specific motor 
abilities of upper and lower limb, as investigated by BBS, Trunk control test, Motricity indices and MAS. For this reason these last scales, in our opinion, are better suited for investigating motor aspects of hemiplegics in the early postacute phase.

Our data are interesting because of the homogeneity of the population studied and the short time interval considered in measuring the outcomes. They confirm, in a selected population of early post acute stroke patients, the findings published on more heterogenic patients' populations in previous researches. For example, Lohse et al. (9) found a positive relationship between time scheduled for therapy and outcomes with mean time post stroke of one year, and Veerbeek et al. (19), who investigated the effects of different interventions on different functional measures (i.e. the effects on gait, arm/hand function, balance), also found positive results. Previous studies, on the other hand, revealed small benefits of the therapy dose, for example the study of Kwakkel et al. who reported nearly 0.020 correlation with measures of ADLs and walking speed (20). However in Kwakkel et al.'s meta-analysis, the authors did not accurately measure the difference in treatment duration between patients. A recent study also reported that convalescent stroke patients, who increased their number of physiotherapies from 3 to 6 hours/day, demonstrated improvement in their walking ability, especially those with complete paralysis or severe paralysis of the lower limbs. However, the population studied in this research was examined after a mean of one-month post stroke (21). Recently a Canadian review analyzing the results of eight randomized clinical trials comparing stroke patients, examined from 13 to 42 days (mean) from stroke, receiving high or low intensity physiotherapy, revealed that higherintensity upper-limb and higher-intensity physiotherapy both resulted in significantly greater improvements in motor function. The results on the effect of physiotherapy also involved the chronic post stroke phase (8).

The main limitation of our study was the absence, due to ethical reasons and to the observational nature of the study itself, of a control group of patients, who did not receive any physiotherapic intervention. This issue, which is relevant in order to separate the effects of spontaneous recovery from the role of physiotherapy, can, in our opinion, hardly be overcome in future research. Furthermore, the absence of imaging data, in order to control for the effects of lesion size on stroke evolution, is another limitation. Due to the retrospective nature of the study, analyzing the clinical data on available records of stroke patients, the presence of missing data can be regarded as a limit as well. Finally, the absence of information about neuropsychological deficits of each patient is worth to be considered as a defect, in particular the presence or absence of language, spatial exploration, memory or orientation deficits, which could have been responsible, at least in part, for differential outcomes in daily activities. Particularely, Gialanella and Mattioli found that anosognosia, and nonunilateral spatial neglect, is the worst prognostic factor for motor recovery in right brain damaged stroke patient (22).

The presented results of a greater motor improvement in patients, who received greater physiotherapy load can be interpreted in terms of increased plasticity due to exercise. Studies on animals and humans have demonstrated that large quantities of practice lead to cortical reorganization and improved behavioral function. Similar studies linked neural changes with recovery of function and learning in adults post-stroke $(23,24)$. These data indicate that increased practice leads to greater skills, as long as practice is challenging, progressive and skill-based (23-25).

The direct relationship between the amount of physiotherapy provided in the early post stroke phase and the degree of motor amelioration, suggests that providers of rehabilitation services should consider an increase in therapy time for early post acute stroke patients. Although, future studies will be needed in order to systematically compare the effects of high versus low intensity physiotherapies in early post acute stroke patients, our data support the observation that higher-intensity physiotherapy may result in lower costs and improved health outcomes (11).

Implications of the manuscript: A significant correlation between the amount of physiotherapy and the improvement in many functional scales in early post stroke patients was shown in this retrospective real life study. This result prompts further randomized clinical trials comparing different groups of patients receiving different amounts of physiotherapy, and suggests treatment of early post stroke patients with intensive motor rehabilitation.

\section{Acknowledgments}

We acknowledge all the physiotherapists, who helped with the data collection.

\section{Footnote}

Authors' Contribution: Caterina Sottini developed the original idea, Daniela Vangi and Emanuela Rizzi contributed to data collection, Chiara Stampatori analyzed data, Flavia Mattioli wrote the manuscript.

\section{References}

1. Roger VL, Go AS, Lloyd-Jones DM, Adams RJ, Berry JD, Brown TM, et al. Heart disease and stroke statistics-2011 update: a report from the American Heart Association. Circulation. 2011;123(4):e18-e209. doi: 10.1161/CIR.ob013e3182009701. [PubMed: 21160056]. 
2. Wardlaw JM, Del Zoppo GJ, Yamaguchi T, Berge E. Thrombolysis for acute ischaemic stroke. Cochrane Lib. 2003.

3. Stroke Unit Trialists C. Organised inpatient (stroke unit) care for stroke. Cochrane Database Syst Rev. 2007(4):CD000197. doi: 10.1002/14651858.CD000197.pub2. [PubMed: 17943737].

4. Wade DT, Hewer RL. Functional abilities after stroke: measurement, natural history and prognosis. J Neurol Neurosurg Psychiatry. 1987;50(2):177-82. [PubMed:3572432].

5. Miller EL, Murray L, Richards L, Zorowitz RD, Bakas T, Clark P, et al. Comprehensive overview of nursing and interdisciplinary rehabilitation care of the stroke patient: a scientific statement from the American Heart Association. Stroke. 2010;41(10):2402-48. doi: 10.1161/STR.ob013e3181e7512b. [PubMed: 20813995].

6. IONIO . ISTAT database 2013. Available from: http://ionio.cineca.it/.

7. Chow DS, Hauptman JS, Wong TT, Gonzalez NR, Martin NA, Lignelli AA, et al. Changes in stroke research productivity: A global perspective. Surg Neurol Int. 2012;3:27. doi: 10.4103/2152-7806.92941. [PubMed: 22439118].

8. Sehatzadeh S. Effect of Increased Intensity of Physiotherapy on Patient Outcomes After Stroke: An Evidence-Based Analysis. Ont Health Technol Assess Ser. 2015;15(6):1-42. [PubMed: 26356355].

9. Lohse KR, Lang CE, Boyd LA. Is more better? Using metadata to explore dose-response relationships in stroke rehabilitation. Stroke. 2014;45(7):2053-8. doi: 10.1161/STROKEAHA.114.004695. [PubMed: 24867924].

10. Kreisel SH, Bazner H, Hennerici MG. Pathophysiology of stroke rehabilitation: temporal aspects of neuro-functional recovery. Cerebrovasc Dis. 2006;21(1-2):6-17. doi: 10.1159/000089588. [PubMed: 16282685].

11. Chan B. Effect of Increased Intensity of Physiotherapy on Patient Outcomes After Stroke: An Economic Literature Review and CostEffectiveness Analysis. Ont Health Technol Assess Ser. 2015;15(7):1-43. [PubMed: 26366241].

12. van Swieten JC, Koudstaal PJ, Visser MC, Schouten HJ, van Gijn J. Interobserver agreement for the assessment of handicap in stroke patients. Stroke. 1988;19(5):604-7. [PubMed: 3363593].

13. Collin C, Wade DT, Davies S, Horne V. The Barthel ADL Index: a reliability study. Int Disabil Stud. 1988;10(2):61-3. [PubMed: 3403500].

14. Carr JH, Shepherd RB, Nordholm L, Lynne D. Investigation of a new motor assessment scale for stroke patients. Phys Ther. 1985;65(2):17580. [PubMed: 3969398].

15. Collin C, Wade D. Assessing motor impairment after stroke: a pilot reliability study. J Neurol Neurosurg Psychiatry. 1990;53(7):576-9.
[PubMed: 2391521].

16. Viosca E, Martinez JL, Almagro PL, Gracia A, Gonzalez C. Proposal and validation of a new functional ambulation classification scale for clinical use. Arch Phys Med Rehabil. 2005;86(6):1234-8. doi: 10.1016/j.apmr.2004.11.016. [PubMed: 15954065].

17. Franchignoni FP, Tesio L, Ricupero C, Martino MT. Trunk control test as an early predictor of stroke rehabilitation outcome. Stroke. 1997;28(7):1382-5. [PubMed: 9227687].

18. Berg K, Wood-Dauphine S, Williams JI, Gayton D. Measuring balance in the elderly: preliminary development of an instrument. Physiother Canada. 1989;41(6):304-11.

19. Veerbeek JM, van Wegen E, van Peppen R, van der Wees PJ, Hendriks E, Rietberg M, et al. What is the evidence for physical therapy poststroke? A systematic review and meta-analysis. PLoS One. 2014;9(2):ee87987. doi: 10.1371/journal.pone.0087987. [PubMed: 24505342].

20. Kwakkel G, van Peppen R, Wagenaar RC, Wood Dauphinee S, Richards C, Ashburn A, et al. Effects of augmented exercise therapy time after stroke: a meta-analysis. Stroke. 2004;35(11):2529-39. doi: 10.1161/01.STR.0000143153.76460.7d. [PubMed: 15472114].

21. Kawakami K, Tanino G, Tomida K, Kato Y, Watanabe M, Okuyama Y, et al. Influence of increased amount of exercise on improvements in walking ability of convalescent patients with post-stroke hemiplegia.JPhys Ther Sci. 2016;28(2):602-6. doi:10.1589/jpts.28.602. [PubMed: 27065551].

22. Gialanella B, Mattioli F. Anosognosia and extrapersonal neglect as predictors of functional recovery following right hemisphere stroke. Neuropsychol Rehabil. 1992;2(3):169-78.

23. Boyd LA, Vidoni ED, Wessel BD. Motor learning after stroke: is skill acquisition a prerequisite for contralesional neuroplastic change?.Neurosci Lett. 2010;482(1):21-5. doi:10.1016/j.neulet.2010.06.082. [PubMed: 20609381]

24. Schaechter JD, Fricker ZP, Perdue KL, Helmer KG, Vangel MG, Greve $\mathrm{DN}$, et al. Microstructural status of ipsilesional and contralesional corticospinal tract correlates with motor skill in chronic stroke patients. Hum Brain Mapp. 2009;30(11):3461-74. doi:10.1002/hbm.20770. [PubMed: 19370766].

25. Taub E, Uswatte G, Mark VW, Morris DM, Barman J, Bowman MH, et al. Method for enhancing real-world use of a more affected arm in chronic stroke: transfer package of constraint-induced movement therapy. Stroke. 2013;44(5):1383-8. doi: 10.1161/STROKEAHA.111.000559. [PubMed: 23520237]. 